\title{
Poster Abstracts (Session 3)
}

(Full Posters are available at http://www.journals.cambridge.org/jid_IAU)

\section{Abundances from Disentangled Component Spectra: High-mass Stars in the Eclipsing Binaries CW Cep and V478 Cyg}

K. Pavlovski and E. Tamajo

Department of Physics, University of Zagreb, Zagreb, Croatia

Techniques for the spectral disentangling of the composite spectra of close binaries, in combination with light-curve analysis, make it possible to reconstruct individual component spectra. In an on-going project we are making photospheric elemental abundance determinations for high-mass stars in OB binaries. The principal goal of the project is an observational test of rotational evolutionary stellar models of high-mass stars, and calibration of the photospheric abundance changes in terms of the stellar mass, the fractional life-time on the Main Sequence, and the rotational velocity.

In the present work two more OB binaries, CW Cep and V478 Cyg, with masses of the components in the range of $12-15 \mathrm{M}_{\odot}$ were analyzed. Results would be discussed in the context of our previous studies in this series, and compared to the theoretical predictions.

\section{Accretion Disks of Binary Stars as a Probe of Accretion Disks in Other Astrophysical Objects}

\author{
R.N. Eze \\ University of Nigeria, Nsukka, Enugu, Nigeria
}

The understandings of the processes that lead to accretion in astrophysical objects have been a central problem for some decades now. However, we have a more detailed knowledge of binary disks accretion process because, first there are large number of such systems within $100 \mathrm{pc}$; and such proximity allows fainter spectral features to be analyzed. Secondly, the primary star in a binary can sometimes be used as probe, eclipsing portions of the disks at different times, thereby allowing disks properties to be mapped. Finally, accretion disks occur over a time scale convenient for observational monitoring and much can be leaned by studying the evolving spectra. In this work an attempt has been made to use the results from spectral analyses of the accretion disks of cataclysmic variables to make generalization on accretion disks of other astrophysical objects. For the 15 cataclysmic variables studied there is sufficient evidence to show that their accretion disks are highly ionized. This result supports the fact that the angular momentum transport which lead to accretion is purely by magneto rotational instability if the accretion disks is adequately ionized. 


\section{A Potential Stabilizing Magnetic Effect for Astrophysical Jets}

M.V. Viallet and H. Baty

Astronomical Observatory, Strasbourg, France

Observations show that astrophysical jets are able to propagate over huge distances in comparison with their radial extents. This challenges theoretical and numerical studies, which predict a fast disruption of the jet by the development of internal magnetohydrodynamic instabilities. Particularly important is the Kelvin-Helmoltz instability, known to be the most dangerous for the jet's collimation. Here we use the modern finite-volume based MHD code, VAC, to reinvestigate the development of the Kelvin-Helmholtz instabilities by using high-resolution spatial simulations. We show that an initial large-scale disruption occurs after a propagation distance of a few tens of jet radius, in agreement with previous studies. In addition, we establish the existence of a new mechanism driven by the initial disruption of the jet. This mechanism gives rise to a local reinforcement of the magnetic field, which in turn leads to a new more stable configuration of the jet.

\section{Comparison of Different Spectral Disentangling Techniques Applied to a Triple System}

K.B.V. Torres ${ }^{1,2}$, L.P.R. Vaz ${ }^{1}$, and H. Hensberge ${ }^{2}$

${ }^{1}$ Dept. de Fisica, ICEx, Universidade Federal de Minas Gerais, Belo Horizonte, Brazil,

${ }^{2}$ Royal Obervatory of Belgium, Brussels, Belgium

Spectral disentangling allows us to reconstruct the contribution of multiple stars to a composite spectrum. Different algorithms have been proposed, which fall in two categories: algorithms operating on the Fourier components of the spectra and algorithms operating on the velocity bins (logarithm of wavelength). Almost all applications use the former algorithms because of public availability of software code and the computing time involved. Nevertheless, the simultaneous use of both techniques would give a better insight in the error budget, since each algorithms is sensitive in a different way to uncertainties in the input data and the options available to assign weights to the input data are different.

We will discuss the application of both techniques to a triple system, consisting of a dominant stationary third component dominating the composite spectrum and a close binary with one component much fainter than the other. Several spectral regions are selected, some of which contain telluric lines. When necessary for a better understanding, simpler artificial spectra are also analysed by both techniques. 


\section{An Expanded Bandpass List for Atmospheric Emission in Eclipsing Binary Models}

W. Van Hamme ${ }^{1}$ and R. E. Wilson ${ }^{2}$

${ }^{1}$ Department of Physics, Florida International University, Miami, FL, USA,

${ }^{2}$ Astronomy Department, University of Florida, Gainesville, FL, USA

Programs for modeling binary star observables compute emergent intensity for a given composition as it varies with local effective temperature, local gravity, and direction. With the arrival of huge data sets from Gaia and other surveys, the benefits of fast, compact, and accurate computation of atmospheric radiation is likely to remain critical for the foreseeable future. Experience has shown that accurate radiative modeling is important for good parameter estimation. Here we augment the radiative treatment by Van Hamme \& Wilson (2003, ASP Conf. Ser. 298, Gaia Spectroscopy, Science and Technology, ed. U. Munari (San Francisco: ASP), 323) with a procedure by which individuals can generate the needed Legendre coefficients for arbitrary photometric bands. Resulting files can be inserted directly into the Wilson-Devinney program without sacrifice of portability or program unity, and should easily be adaptable to other binary star programs. We expect the new bandpass options to become part of the public W-D program. Limb-darkening

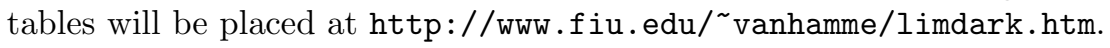

\section{HD 61273: a New Semi-Detached Binary with an Accretion Disk}

F. Royer ${ }^{1}$, P. North ${ }^{2}$, D. Briot ${ }^{1}$, G. Burki ${ }^{3}$, and F. Carrier ${ }^{4}$

${ }^{1}$ Observatoire de Paris, Meudon, France,

${ }^{2}$ Laboratoire d'Astrophysique - EPFL, Sauverny, Switzerland,

${ }^{3}$ Observatoire de Genève, Sauverny, Switzerland,

${ }^{4}$ Instituut voor Sterrenkunde, Leuven, Belgium

A detailed spectroscopic and photometric analysis shows for the first time that HD 61273 is a close binary system, composed of a dwarf early-type star and a K0 giant.

The system underwent and perhaps still undergoes a mass transfer. The most evolved star fills its Roche lobe and transfers mass to the hotter companion which is now the most massive component of the system. The $\mathrm{H} \alpha$ line displays a variable emission: signature of an accretion disk. This new semi-detached system is not eclipsing, but only shows photometric ellipsoidal variability due to the elongated shape of the giant component filling its Roche lobe, with a 12.919 day period. 


\section{Doppler Imaging of Stars with Roche-Geometry}

Zs. Kövári ${ }^{1}$, J. Bartus ${ }^{2}$, K. Oláh ${ }^{1}$, K.G. Strassmeier ${ }^{2}$, J.B. Rice ${ }^{3}$, M. Weber ${ }^{2}$, and

E. Forgács-Dajka ${ }^{4}$

${ }^{1}$ Konkoly Observatory, Budapest, Hungary,

${ }^{2}$ Astrophysical Institute Potsdam, Potsdam, Germany,

${ }^{3}$ Brandon University, Brandon, Canada,

${ }^{4}$ Eötvös University, Dept. of Astronomy, Budapest, Hungary

Tests are carried out on retrieving Doppler maps from distorted stars in close binaries to estimate how Doppler imaging may be aliased by the ellipticity. Maps obtained for the distorted shape are compared with the results of the simple spherical approximation, using real data of the RS Cvn-type close binary star $\zeta$ Andromedae.

\section{Hydrodynamic Simulations of Illuminated Secondary Atmospheres in Dwarf Novae}

M. Viallet and J.M. Hameury

Astronomical Observatory, Strasbourg, France

Dwarf novae (DN) are a subfamily of cataclysmic variable, i.e., binary stellar systems where a white dwarf accretes matter through an accretion disc from a stellar companion. They undergo regular outbursts lasting a few days with a recurrence time of a few tens of days. The outburst phenomenon is best interpreted as being due to a thermal/viscous limit cycle of the accretion disc. The general framework of the disc instability is now well understood, but some points are still unclear. In particular, the possibility that the illumination of the secondary atmosphere leads to an enhancement of the mass transfer rate is still under debate. We present here the first 2D hydrodynamic simulations of the surface flows in the secondary that result from the strong inhomogenous heating of the atmosphere during outbursts. We also discuss the possibility that the L1 point, from which matter leaves the secondary, is directly heated by the disc rim. During an outburst this could in turn contribute to the mass transfer enhancement.

\section{Properties of Circumstellar Dust in Symbiotic Miras}

D. Kotnik-Karuza ${ }^{1}$, T. Jurkic ${ }^{1}$, and M. Friedjung ${ }^{2}$

${ }^{1}$ Department of Physics, University of Rijeka, Croatia,

${ }^{2}$ Institut D'Astrophysique de Paris, Université Pierre \& $\mathcal{S}^{3}$ Marie Curie, Paris, France

We present a study of the properties of circumstellar dust in symbiotic Miras during sufficiently long time intervals of minimal obscuration. The published $J H K L$ magnitudes of o Ceti, RX Pup, KM Vel, V366 Car, V835 Cen, RR Tel and R Aqr have been collected. In order to investigate their long-term variations, we removed the Mira pulsations to correct their light curves. Assuming spherical temperature distribution of the dust in the close neighbourhood of the Mira, the DUSTY code was used to solve the radiative transfer in order to determine the dust temperature and its properties in each particular case. The preliminary results of this systematic study of dust envelopes in symbiotic stars with Miras as cool components provide information on nature of dust in these objects. 


\title{
MIDI Observations of IRCs : Constraining the Geometry of the Warm Circumstellar Environment
}

\author{
S. Correia ${ }^{1}$, Th. Ratzka ${ }^{2}$, G. Duchene ${ }^{3}$, and H. Zinnecker ${ }^{1}$ \\ ${ }^{1}$ Astrophysikalisches Institut Potsdam (AIP), Potsdam, Germany, \\ ${ }^{2}$ MPIA, Heidelberg, Germany, \\ ${ }^{3}$ LAOG, Grenoble, France
}

Despite more than a decade of investigations, the nature of Infrared Companions (IRCs) is still a matter of debate. While the hypothesis that IRCs could be in an earlier evolutionary stage than their primaries implies that they are embedded in an optically thick envelope, recent high spectral resolution near-infrared spectroscopy would rather favor the scenario of IRCs being normal $\mathrm{T}$ Tauri stars seen through an almost edge-on disk. We will report on recent high-spatial resolution interferometric observations of the IRCs Glass-I, Haro 610 and VV CrA obtained in the Mid-IR with MIDI/VLTI which provide further insights into the geometry of their dusty environment and will in general contribute to a better understanding of this intriguing class of objects.

\section{Window to the Stars}

R.G. Izzard and E. Glebbeek

University of Utrecht, Utrecht, Netherlands

We present a graphical user interface to the popular TWIN stellar evolution code. It removes the drudgery associated with the traditional approach to running the code, while maintaining the power, output quality and flexibility a modern stellar evolutionist requires.

\section{Spectral Disentangling and Combined Orbital Solution for the Hyades Binary $\theta^{2}$ Tau}

\author{
P. Lampens, Y. Frémat, P. De Cat, and H. Hensberge \\ Koninklijke Sterrenwacht van Belgie, Brussel, Belgium
}

Theta $^{2}$ Tau is a detached, "single-lined" binary and the most massive resolved spectroscopic binary of the Hyades cluster. It also shows a complex pattern of pulsations of type $\delta$ Scuti. Its eccentric orbit $(\mathrm{e}=0.7)$ has a period of 140.7 days. Component B is very difficult to detect spectroscopically. For this reason, the few radial velocities published in the literature are inaccurate. From recent high-resolution spectroscopic data obtained with the ELODIE spectrograph at the OHP (France), we derived accurate radial velocities for *both* components, applying a spectral disentangling algorithm (by P. Hadrava, 1999). We then combined these measurements with available very-large baseline interferometric data in order to improve the knowledge of the orbital parameters and derived fundamental properties in a self-consistent way. Such determination is also very pertinent to revisit the evolutionary status of both components. 


\section{RR Lyrae in M15: Fourier Decomposition and Physical Parameters}

G. Garcia Lugo ${ }^{1}$, A. Arellano Ferro ${ }^{2}$, and P. Rosenzweig ${ }^{1}$

${ }^{1}$ Universidad de Los Andes, Mérida, Venezuela,

${ }^{2}$ Instituto de Astronomía, Universidad Nacional Autónoma de México, D F, Mexico

In the present study, $V$ and $R$ images of M15 were obtained in two seasons during the year 2000 and 2001, respectively. These images were taken using the 1.5-m telescope of San Pedro Mártir Observatory, in Baja California, México. The telescope was equipped with a CCD Tektronix of $1024 \times 1024$ pixels with a size of $24 \mu^{2}$.

In this work, results of CCD photometry are reported for 33 known RR Lyrae stars in M15. The periodicities of some variables have been recalculated and new ephemeredes are given. The Blazhko effect, reported previously for the V12 star, has not been detected. Through the use of the technique of Fourier decomposition of the light curves, the physical parameters of the type RRab and RRc variables have been estimated. The cluster is Oosterhoff type II and the values determined for the iron content and the distance are $[\mathrm{Fe} / \mathrm{H}]=-1.98 \pm 0.24 \mathrm{y}$ and $\mathrm{d}=8.67 \pm 0.41 \mathrm{kpc}$, respectively. The mean values of the physical parameters determined for the RR Lyrae stars, place the cluster correctly in the sequences Oosterhoff type - metallicity and metallicity - effective temperature, valid for globular clusters. These sequences suggest that the origin of the Oosterhoff dichotomy is of evolutive nature. 